\title{
Sketch-Based Rapid Prototyping Platform for Hardware-Software Integrated Interactive Products
}

\author{
Tek-Jin Nam \\ CIDR (Collaborative \& Interaction Design Research) \\ Dept. of Industrial Design, KAIST, 373-1, Gusung-Dong, \\ Yusung-Gu, Daejeon, 305-701, Korea \\ tjnam@kaist.ac.kr
}

\begin{abstract}
This paper presents a platform in which interaction designers can effectively and rapidly develop tangible interactive prototypes by sketching. The study aims to build a platform that plays the role of sketching in the hardware-software integrated interactive product design process. The platform consists of three components: a sketch based interactive concept exploration software application called STCtools, a set of physical user interface(PUI) widgets with a key converter and a video projection based Augmented Reality desk (ARdesk). For prototyping, a designer creates hardware and software sketches with pen based computers using STCtools. Sketches of hardware and software are drawn in a client device and composed in an electronic whiteboard, which is the server device. PUI widgets can be physically attached on a foam mockup or on a screen of the client device. The hardware-software integrated simulations are conducted on ARdesk. The sketch simulation is captured and projected onto a paper marker created with invisible infra-red ink.
\end{abstract}

\section{Author Keywords :}

Interactive Product Design, Prototyping, Sketch based design tool, Augmented Reality, Interaction Design, Conceptual Design

\section{ACM Classification Keywords}

H.5.2. [User Interfaces]: Prototyping, D.2.2 [Design Tools and Techniques]: Evolutionary prototyping, H.5.1 [Multimedia Information Systems]: Artificial, augmented, and virtual realities

\section{INTRODUCTION}

Sketching is a means of effective communication and creative concept development. Lawson[6] suggested that as a tool of thought, drawing is vital to many designers. Not only does the act of drawing freeze ideas and put them open for examination, but also it actually mediates creative thought processes. Powell[12] mentioned that an industrial designer who cannot draw is certainly less efficient and almost always

Copyright is held by the author/owner(s).

CHI 2005, April 2-7, 2005, Portland, Oregon, USA.

ACM 1-59593-002-7/05/0004. less creative than one who can. For designers, sketching is a tool for idea development, creative thinking and examination. It also serves as a collaborative medium between concept designers and engineers. For all the participants in the design process, it is a powerful communication tool.

When designers are dealing with interactive products, one of the problems is that the traditional sketches cannot cover all aspects. In addition to hardware aspects of a product, consideration should be given to contents, information architecture and user interface as well. The integration of these design elements is difficult without a close collaboration between professionals with different specialties. In designing interactive products, there is a lack of tools that play the role of sketching, as in traditional industrial design projects. In particular, the integration of all the design elements is particularly difficult to achieve in the early phase of the design process when ideas evolve and change rapidly. The effective exploration of integrated design concepts is one of the major challenges in prototyping.

To address these issues, this study proposes a prototyping platform that uses the sketching metaphor. The platform applies Augmented Reality technology and employs sketching as the main interface as well as the metaphor in terms of its role in the design process.

\section{Related Works}

There have been researches on prototyping tools and the application of sketching in prototyping. DENIM[11] is a sketch based website design tool. It supports informal design of information and navigation design. Designers' Outpost[2] combines virtual and real sketches. These studies focused largely on software aspects. Paper prototyping[14] is a useful tool in the early design phase. The focus of paper prototyping is however, on software aspects and user study. Phidgets[3] and Calder toolkits[7] allows designers to explore tangible user interface for interactive product design. However, it is targeted for programmers and engineers. Users without programming and engineering knowledge have difficulties in using these tools. Other professionals who take part in the design process, such as psychologists and social scientists, also need to have interactive prototyping skills sometimes to understand new design problems. 
For designers without low level programming and electronics skills, we suggested the use of PowerPoint and its embedded programming environment[9]. We also suggested that software simulation can be integrated in two Augmented Reality environments [10], with a HMD display and a high resolution video projector. This work however considers the integration for more concretized ideas in later stages of design.

\section{THE PROTOTYPING PLATFORM}

Although design tools are becoming digital, conventional pen and paper based design tools are still widely used by designers because it is effective and intuitive in the conceptual design phase. One important issue to consider is the use of conventional media in accordance with new digital design tools. The familiar visualization methods have to be smoothly combined in the design process. We propose a platform that allows designers to develop ideas of hardware aspects such as the shape of interactive products, the locations of physical user interface elements, software contents and the user interface. It supports the integration of all these elements with sketches from the early phase of the design process.

The platform consists of three components: 1) a sketch based interactive concept exploration software application called STCtools, 2) a set of Physical User Interface (PUI) widgets with a key converter and 3) a video projection based Augmented Reality desk (ARdesk).

\section{STCTOOLS: SKETCH-BASED INTERACTIVE CONCEPT EXPLORATION SOFTWARE APPLICATION}

To explore the organizational and interactive aspects of interactive products, we use State Transition Charts (STC). STC is based on the idea that interaction can be effectively modeled by articulating states and events. STC provide a way of exploring and defining the states that should exist, the events that should occur and the effects they will have on the product.

STC may be similar to State Transition Diagram for software engineering. For working with STD, there have been proprietary or prototype level tools such as visualSTATE [5], SDL[13] and Statecharts [4]. STC is however, more graphical and realistic in terms of the visual components and the associated events of the product. One method that allows more dynamic and collaborative creation of STC is to use Post-it notes and a whiteboard to develop STC [10]. By drawing essential states on the Post-it notes, then placing and connecting the states on the whiteboard, a new STC can be constructed rapidly. STCtools (State Transition Chart Tools) transforms and empowers this approach with pen-based digital technologies.

\section{Three Modules of STCtools: STCmake, STCcompose, and STCrun}

STCtools provides a digital environment for creating, composing and running STC. It is a set of software applications that run on pen-based devices such as PDAs,

Tablet PCs and Desktop PCs with MimioC[8]. It includes three applications: STCmake, STCcompose, and STCrun. The first application, STCmake running on a client device, corresponds to the post-it notes, with which the main task is to create states. When a new state is created in STCmake, it is transferred to the networked application, STCcompose, running on a server device. This action corresponds to putting the Post-it notes on a whiteboard. The states on STCcompose may be moved and rearranged to refine the structure and the flow of the user interface. Event links between the states may be defined in STCcompose. As the arrangements of states and event transition links between the states develop, STC may be tested interactively with the last application, STCrun, running on both the client and server devices.

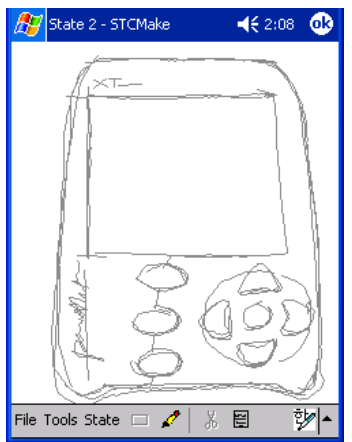

Figure 1. Sample screen of STCmake

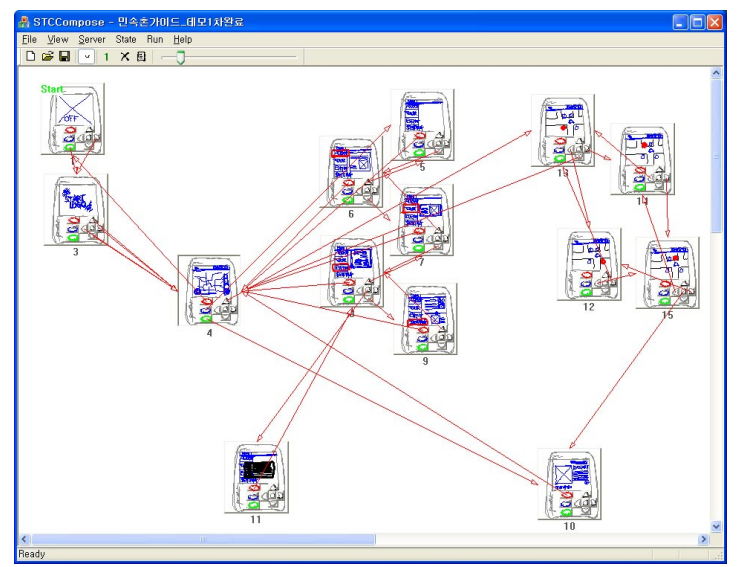

Figure 3. Sample screen of STCcompose

Figure 1 shows a sample screen of STCmake. Basic pen based drawing functions such as draw, select, delete and property selection, are provided in STCmake. STCcompose has a large workspace window in the middle to manipulate and rearrange states (Figure 3). The STCrun (Figure 2) can be launched within STCcompose or separately.

The hardware systems used with STCtools may easily become portable (for example, PDA client + Tablet PC server combination) while other combinations are also possible for different requirements. STCcompose was implemented in Visual $\mathrm{C}++$.net. STCmake and STCrun were implemented in Embedded $\mathrm{C}++3.0$ and Visual 
$\mathrm{C}++$.net. The tested target hardware devices were iPAQ 3870 PDA, Compaq TC1000 Tablet PC and generic windows PC. The network connection was a general wired/wireless Internet and the communication protocol used in the application development was TCP/IP.

\section{Physical User Interface Widgets and a Key Converter}

When designers create sketches of states and events using STCtools, PUI widgets allow designers to explore tangible aspects of interactive products. The second component of the platform is a set of different types of buttons and a key converter (Figure 4). The buttons have thin wires and connectors. The buttons have no electronic components or batteries inside. Reusable adhesives such as UHUtac $([15]$ are attached on the back of the buttons. Therefore, the buttons can be attached and moved to any surfaces including the screen surface of the tablet PC and foam mockups (Figure 5 ). The key converter is used to make simple connections between the buttons and STCtools. It transforms signals from external input widgets to simple keyboard input signals through the PS2 cable. When states are created in STCtools, a simple button press operation can be used to define the events in STCtools. Wireless PUI widgets using RF or IR can be considered as an alternative but the size of the widgets and the additional equipments could be potential constraints.

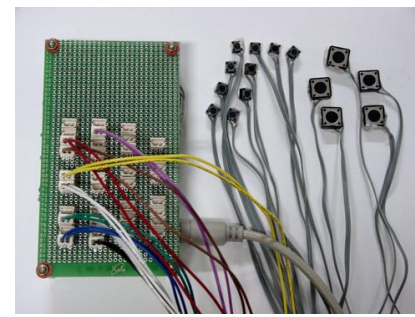

Figure 4. The set of PUI widgets (Buttons \& key converter)

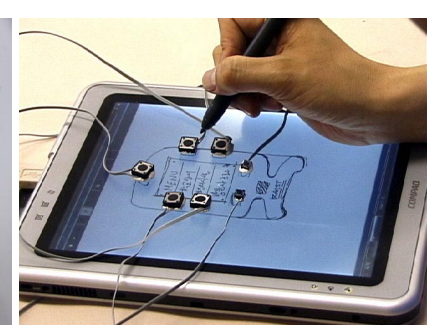

Figure 5. PUI widgets attached on the screen of a Tablet PC

\section{AUGMENTED REALITY DESK (ARDESK)}

It is useful to have a large electronic workspace when using the STCComposer module of STCtools. This workspace is provided on ARdesk. ARdesk is an electronic whiteboard equipped with video projection based Augmented Reality features. It has double video projectors. The back video projection is used for the electronic whiteboard. On the surface of the desk, a pen input device, Mimio $®[8]$, is installed. The front video projection is used to overlay virtual display onto a real paper marker. Different sized paper markers can represent the various display parts of an interactive product. The interactive simulation created in STCtools with sketches are captured and projected onto the paper markers. The front projection overlays the display onto the marker that may be attached to foam mockups. When a foam mockup with a maker is located on the ARdesk, users can touch and operate PUI while the display of state sketches changes interactively according to the PUI operations. ARdesk supports seamless transition between state

compositions and the tangible usage experience of the intermediate or final results.

To build a more compact configuration of the ARdesk, tracking and video projection was installed on the front side. The projector and the camera for marker detection was integrated and attached to a single tripod. The captured screen image of STCrun is projected onto the paper marker while the camera detects the identity and location of the marker. The invisible paper marker was made with infrared ink. The camera with an infrared filter could detect the marker pattern without interfering the projected video image on the surface of the paper marker. We used ATtoolkit[1] for marker tracking.

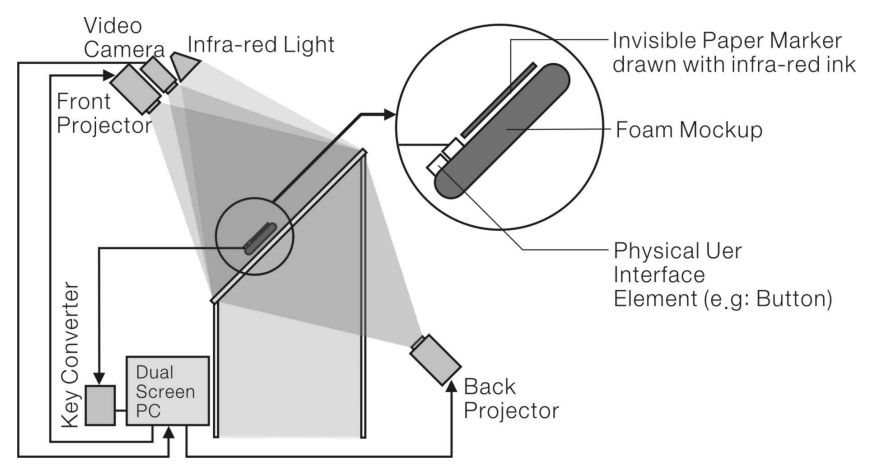

Figure 6. Configuration of ARdesk

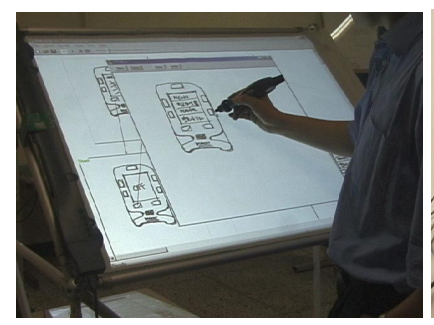

Figure 7. Composing states in STCtools

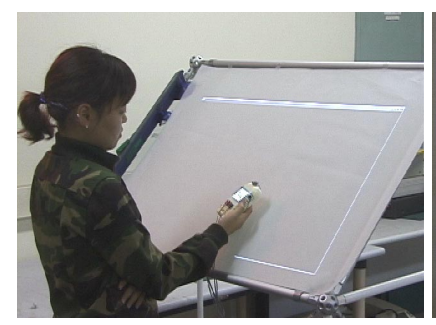

Figure 9. testing Integrated simulation on ARdesk

\section{USAGE SCENARIO}

The usage scenario of the prototyping platform can be divided into several steps. In the first step, a designer creates hardware sketches using STCmake. Sketches of contents and user interface concepts are also created. The states are transferred, composed in STCcomposer workspace on ARdesk (Figure 7). Related event links are added. The PUI widgets (buttons) are attached on the surface of the device 
running STCmake, while states are created and refined (Figure 8). In the following step, the designer examines and tests simulations of the composed states on the tablet PC or on a foam mockup. Next, the designer examines the simulations on the ARdesk to combine the display of sketch simulation and the foam mockup (Figure 9, 10). The designers refine and finalize the intermediate results by iteratively repeating these steps.

\section{PRELIMINARY USER STUDY}

A formal and structured user study is in progress. A preliminary informal user study was carried out in a design workshop with university students. The design sessions were video recorded for analysis. The project was to design a portable campus tour digital guide. The results showed that the participants quickly learnt and understood the role of the platform components. The design process was found to be effective in this relatively simple design project. The platform seemed to help iterative and evolutionary concept development. It was found that with the interactive simulation, the number of states and events were rapidly increased during the workshop. The participants frequently tested the intermediate results.

Some technical problems were also found. The video resolution of the projected screen became a problem when the size of the display became small. When users moved the mockup towards the projector, the augmented video images went out of focus. Therefore the area that the users can overlay the video display onto the foam mockup was limited. The shadow of the user sometimes blocked the front video projection. Nevertheless, feedbacks from the participants were very positive. Participants particularly liked the fact that the platform allows them to see the interactive results as they developed new ideas, even though it was in a sketch form.

\section{CONCLUSION}

This paper presents a prototyping platform to assist designing hardware software integrated interactive products. The platform can be further refined as a designers' workbench where effective prototyping can be carried out in the concept design stage. There are a number of directions for future work. A comparison with traditional methods may provide some insights on the application of this platform. Improvements should also be made to STCtools in terms of the drawing and editing states with pen based devices. Methods incorporating various hardware interface concepts should be considered. More structured user study should be carried out to understand the impact of the new prototyping platform on communication and idea deployment aspects in designing interactive products. This may require a field study with real designers. The prototyping should also be incorporated within the design cycle of user interface development.

\section{REFERENCES}

1. Billinghurst M.and H. Kato, "Collaborative Mixed Reality", Proc ISMR'99, Yokohama, Japan, 1999

2. Everitt, K., Klemmer, S., Lee, R. and Landay, J. "Two Worlds Apart: Bridging the Gap Between Physical and Virtual Media for Distributed Design Collaboration" Proc CHI2003, 553-560.

3. Greenberg, S. and Fitchett, C. "Phidgets: easy development of physical interfaces through physical widgets", Proc of UIST2001

4. Harel, D., "Statecharts: a visual Formalism for complex systems", The Science of Computer Programming, 1987, 231-274

5. IAR, "IAR visualSTATE for Embedded Systems", http://www.iar.com

6. Lawson, B. How Designers Think: The Design Process Demystified, Third Edition, Architectural Press, 1997

7. Lee, J., Avrahami, D., Hudson, S., Forlizzi, J., Dietz, P. and Leigh, D. "The Calder toolkit: wired and wireless components for rapidly prototyping interactive devices", in Proc. of DIS2004, 167 - 175

8. Mimio: http://www.mimio.com

9. Nam, T-J. and Lee, W. "Integrating Hardware and Software: Augmented Reality Based Prototyping Method for Digital Products" Proc CHI2O03 Extended Abstract $\mathrm{CD}$

10. Nam, T., Designing Information Appliances: the evaluation of a design process framework based on a designer-friendly prototyping environment, Durling, D. and Shakelton, J. (eds.) Proc. Of Design Research Socety International conference (commonground), 2002

11. Newman, M., Lin, J., Hong, J. and Landay, J. "DENIM: An Informal Web Site Design Tool Inspired by Observations of Practice." In Human-Computer Interaction, 2003, 18(3), 259-324.

12. Powell D. Presentation Techniques: A guide to drawing and presenting design ideas, Macdonald Orbis Book, 1985

13. SDL, "What is SDL", http://www.sdl-forum.org/SDL

14. Snyder, C. Paper Prototyping: The Fast and Easy Way to Design and Refine User Interfaces, Morgan Kaufmann, 2003

15. UHUtac: http://www.uhu.de 